\title{
Elisabeth Rowe Lichttherapie in der Hautarztpraxis - Top oder Flop?
}

\author{
Light Treatment in the Dermatological Practice - Top or Flop?
}

\section{Zusammenfassung}

In deutschen Hautarztpraxen gehörte die Lichttherapie jahrelang zum therapeutischen Standard. Wegen der schlechten Vergütung können es sich immer weniger Hautärzte leisten, die Lichttherapie in ihren Praxen uneingeschränkt anzubieten. Hautärzte sollten Strategien entwickeln, sich die Lichttherapie nicht durch andere Mitbewerber im Gesundheitsmarkt aus den Händen nehmen zu lassen.

\section{Abstract}

Irradiation with ultraviolet light was a therapeutical standard procedure in German dermatological practices for many years. Because of the now rather poor reimbursement the number of dermatologists who can afford offering light treatment without limitations steadily declines. Dermatologists should develop strategies to prevent the takeover of light treatment by other competitors on the health market.
Die Lichttherapie war jahrelang eine wichtige Säule sowohl im Therapiespektrum als auch als Einnahmequelle in dermatologischen Praxen. Dank immer ausgefeilterer technischer Finessen ist es heute möglich, eine Vielzahl von Hautkrankheiten mit maßgeschneidertem Licht zu behandeln. Gehörte die Lichttherapie bis vor einigen Jahren noch zum Standard in fast jeder Hautarztpraxis, zeichnet sich jetzt ein Trend ab, auf diese bewährte Therapieform zu verzichten. Grund für diesen Rückzug ist in erster Linie die schlechte Honorierung ärztlicher und technischer Leistungen. Jeder niedergelassene Vertragsdermatologe, der auch nur ansatzweise betriebswirtschaftlich denkt, kann sich ausrechnen, mit wie vielen GKV-Patienten, die drei- bis viermal in der Woche Lichttherapie konsumieren, sein Budget ausgeschöpft ist. Hinzu kommen Kosten für Strom, Vermessung und Wartung der Geräte. Aus Opportunitätsgründen wird sich der niedergelassene Dermatologe überlegen müssen, ob er die Lichttherapie weiterhin verordnet, selbst anbietet und dafür auf andere Leistungen verzichtet oder ob er zugunsten anderer Leistungen die Lichttherapie einschränkt, gänzlich aus einem Leistungsangebot streicht und Patienten, die dieser Therapie bedürfen, vielleicht schon gute Erfahrungen damit hatten und diese Thera- pie wünschen, an andere Dermatologen, Kliniken, kommerzielle Therapiezentren oder Solarien verweist.

In diesem Artikel soll eine Lanze für die Lichttherapie in der Praxis des niedergelassenen Hautarztes gebrochen werden.

\section{Was spricht für die Lichttherapie?}

- Es handelt sich um eine unbestritten wirkungsvolle und nebenwirkungsarme Therapie. Die häufigste und fast immer unvermeidliche Nebenwirkung ist die Bräunung der Haut, die von fast allen Patienten als sehr angenehm empfunden wird.

- Die Patienten sind in der Regel zufrieden und fühlen sich an die Praxis gebunden.

Dazu trägt wesentlich der fast tägliche Praxisbesuch als soziale Kontaktmöglichkeit bei.

- Der Personalaufwand ist gering. Der vom Arzt aufgestellte Therapieplan wird über drei bis vier Wochen abgearbeitet. Wenn keine besonderen Vorkommnisse auftreten, wird ärztliche Arbeitszeit kaum in Anspruch genommen. 
Tab. 1 Geräteübersicht nach Indikation und Wirtschaftlichkeit

\begin{tabular}{|c|c|c|c|c|c|c|c|c|c|}
\hline Gerät & Indikation & $G K V$ & Pkt & Pkte / Serie & $S Z$ & $€$ & $€ /$ Serie & Investition & Platz \\
\hline UVB $311 \mathrm{~nm}$ & $\begin{array}{l}\text { Psoriasis } \\
\text { Neurodermitis }\end{array}$ & $\begin{array}{l}+ \\
+\end{array}$ & 100 & 2000 & $\begin{array}{l}+ \\
+\end{array}$ & 9,55 & 191,00 & ca. $10000 €^{*}$ & $+++(+)$ \\
\hline UVA (PUVA) & $\begin{array}{l}\text { Psoriasis } \\
\text { Neurodermitis }\end{array}$ & $\begin{array}{l}+ \\
+\end{array}$ & 160 & 3200 & $\begin{array}{l}+ \\
+\end{array}$ & 12,59 & 251,80 & ca. $10000 €^{*}$ & $+++(+)$ \\
\hline $\begin{array}{l}\text { UVA-1-Kalt- } \\
\text { Licht }\end{array}$ & $\begin{array}{l}\text { Neurodermitis } \\
\text { Uveitis } \\
\text { T-Zell-Lymphom } \\
\text { Akne } \\
\text { Mastozytose }\end{array}$ & $\begin{array}{l}- \\
-\end{array}$ & & & $\begin{array}{l}+ \\
+\end{array}$ & 52,46 & $1.049,20$ & ca. $40000 €$ & ++++ \\
\hline BPT & $\begin{array}{l}\text { Psoriasis } \\
\text { Neurodermitis }\end{array}$ & $\begin{array}{l}- \\
-\end{array}$ & & & $\begin{array}{l}+ \\
+\end{array}$ & 32,70 & 654,00 & ca. $40000 €$ & ++++ \\
\hline Lichtschirm & Winterdepression & - & & & + & 9,55 & 191,00 & ca. $600 €$ & + \\
\hline Red'n blue & Akne & - & & & - & 9,55 & 191,00 & ca. $200 €$ & + \\
\hline PDT & $\begin{array}{l}\text { Aktinische Keratose } \\
\text { Präkanzerose } \\
\text { Warzen }\end{array}$ & $\begin{array}{l}- \\
- \\
-\end{array}$ & & & $\begin{array}{l}+ \\
+ \\
+\end{array}$ & 104,83 & & ca. $10000 €$ & ++ \\
\hline Bioptron & Wundheilung & - & & & + & 9,55 & 95,50 & ca. $300 €$ & + \\
\hline IR-A & $\begin{array}{l}\text { Rheuma } \\
\text { Blutdruckregulation } \\
\text { Sklerodermie }\end{array}$ & $\begin{array}{l}- \\
- \\
-\end{array}$ & & & $\begin{array}{l}+ \\
+ \\
+\end{array}$ & 52,46 & $1.049,20$ & ca. $90000 €$ & ++++ \\
\hline UV-C & Arterielle DBS & - & & & + & 25,64 & 256,40 & ca. $1250 €$ & + \\
\hline WIRA & Warzen & - & & & + & 9,55 & 95,50 & са. $1900 €$ & ++ \\
\hline
\end{tabular}

* = als Kombinationsgerät möglich; + = wenig Platzbedarf bzw. transportabel; ++ = Platzbedarf, jedoch transportabel;

$+++=$ Platzbedarf, Patient muss stehen, nicht transportabel; ++++ = Platzbedarf, Patient muss liegen, nicht transportabel

- Da eine Lichttherapie mit mindestens 100 EBM-Punkten pro Sitzung und 2000 Punkten pro GKV-Patient (angenommen 20 Sitzungen) zu Buche schlägt, ist das Budget schnell aufgefüllt. Dieser Vorteil kann allerdings auch zum Nachteil gereichen. Für die übrigen Patienten bleiben weniger Budgetpunkte übrig.

- Die Lichttherapie lässt sich im Selbstzahlersegment einer Praxis gut verkaufen. Aufgrund der vielfältigen Einsatzmöglichkeiten gibt es etliche Patienten, die selbst Beschwerden haben, welche mittels Lichttherapie zumindest gelindert werden können oder sie kennen jemanden, der solche Beschwerden hat. Insbesondere werden auch Patienten angesprochen, die „Kortison“ und „Chemie“ strikt ablehnen und sich zu „natürlichen" Heilverfahren hingezogen fühlen.

- Im Zuge der Aufhebung des Werbeverbotes kann mit der Spezialisierung „Lichttherapie“ unter Berücksichtigung des jeweiligen Kammerrechtes geworben werden.

- Aufgrund der Denkgewohnheiten der Bevölkerung wird die Lichttherapie meist dem Hautarzt zugeordnet. Diesen Vorsprung sollten sich die Hautärzte nicht nehmen lassen und sich für alles zuständig fühlen, was mit Licht und dessen Wirkung, insbesondere an der Haut, aber auch an anderen Organen zu tun hat. Es ist nicht zu übersehen, dass Ärzte anderer Fachgebiete, Zahnärzte, Heilpraktiker, Apotheker und natürlich Solarienbetreiber die therapeutische Nutzung des Lichtes inzwischen entdeckt haben und auch damit werben.

\section{Was spricht dagegen?}

- Das Praxisbudget ist schnell ausgeschöpft.

- Kosten für Investition, Brenner, Strom, Vermessung und Wartung der Geräte

- Raumbedarf

Wie in fast allen Bereichen der Wirtschaft und der Dienstleistungsunternehmen, zu denen unbestritten auch Arztpraxen gehören, zeichnet sich zunehmend eine Spezialisierung und Konzentration auf bestimmte Leistungen ab. Der aufgeklärte Patient erkundigt sich immer öfter danach, wer für sein individuelles Problem besonders kompetent ist. Das sollte für Krankheiten der Haut und für die Lichttherapie immer der Hautarzt sein.

Sicher ist es aus finanziellen und räumlichen Gründen meist nicht möglich, sich ad hoc Geräte für alle möglichen Lichttherapien anzuschaffen (s. Tab.1). Für die Auswahl der Geräte kann es hilfreich sein, über einen gewissen Zeitraum zu analysieren, für wie viele Patienten welche Art der Lichttherapie geeignet wäre. In eine solche Analyse muss auch einfließen, inwieweit es sich dabei um Privatpatienten handelt. Aber auch GKV-Patienten sollten befragt werden, ob sie bereit wären, für bestimmte Therapien eigenes Geld zu investieren. Aufgrund der Klientel der meisten Hautarztpraxen sind für den Einstieg in die Lichttherapie Geräte zur Behandlung von Psoriasis und Neurodermitis gefolgt von Geräten zur Behandlung aktinischer Keratosen und Präkanzerosen und Warzen am wichtigsten. UVB $311 \mathrm{~nm}$, PDT und wIRA decken diesen Bereich mit einem Investitionsvolumen von etwa 22.000,00 € ab. Sollten es die räumlichen Möglichkeiten zulassen, sind für die Ganzkörpertherapie mit UVB 311nm und UVA Geräte zu bevorzugen, in denen der Patient liegen kann. 
Es ist zu überlegen, ob es sinnvoll ist, die Lichttherapie in ein Institut in unmittelbarer Praxisnähe zu verlagern. Steuer- und Unternehmensberater halten diesbezügliche Modelle bereit. Für kassen- und privatärztliche Leistungen können die Geräte von der Hautarztpraxis gemietet werden. Es ist darauf zu achten, dass die UV-Therapie dann in Anwesenheit des Arztes erfolgen muss. Abgerechnet wird nach EBM oder GOÄ. Dienstleistungen des Institutes, die der Arzt lediglich empfiehlt und deren Inanspruchnahme ganz in der Entscheidung des Patienten bzw, Kunden liegen, werden nicht per EBM oder GOÄ abgerechnet. Hier ist das Institut in seiner Preisgestaltung frei und kann auch besondere Angebote machen. Der Kunde hat natürlich auch die Möglichkeit, und darauf sollte er aus Fairnessgründen immer hingewiesen werden, sich einen anderen Anbieter für seinen ärztlicherseits empfohlenen Lichtkonsum auszusuchen.

Wer sich für die Lichttherapie in seiner Praxis entscheidet, ist gut beraten, mögliche Kooperationspartner in der Umgebung der Praxis anzusprechen. Hierfür eigenen sich beispielsweise Rundschreiben an die ärztlichen und zahnärztlichen Kollegen, aber auch an Apotheken, Heilpraktiker, Kosmetikerinnen und Physiotherapeuten. Wirkungsvoller ist es wahrscheinlich, die Vertreter der entsprechenden Zielgruppen nach Praxisschluss zu einem Imbiss einzuladen und über die Lichttherapie $\mathrm{zu}$ informieren. Dabei ergeben sich bestimmt noch andere Möglichkeiten der Kooperation.

\section{Buchbesprechung}

\section{Wundauflagen für die Kitteltasche}

A. Vasel-Biergans, W. Probst

Stuttgart: Wissenschaftliche Verlagsges., 2003. 440 S., 154 farbige Abb., Kart. 22,- . ISBN 3-8047-2003-X

Bei dem vorliegenden Buch handelt es sich um die sorgfältige $\mathrm{Zu}$ sammenstellung der derzeit auf dem Markt befindlichen Wundversorgungsprodukte. Neben einer Kurzbeschreibung der Charakteristika der Wundauflagengruppen bzw. Wundversorgungssysteme stehen die Produktmonographien, die auch eine kleine Auswahl lokal applizierbarer Arzneimittel berücksichtigen, im Mittelpunkt des Buches. Die Monographien beinhalten Angaben zur Wirkungsweise der Verbände sowie deren Indikationsgebiete und Anwendungsweise. Weiterhin wird auf die Zusammensetzung der Produkte und Verpackungseinheiten eingegangen. Produktpreise werden nicht aufgeführt.

Neben Ärzten und Pflegepersonal richtet sich das Buch an alle Berufsgruppen, die an der Wundversorgung beteiligt sind. Durch die kleine handliche Form ist es als Kitteltaschenbuch gut geeignet. Die farbliche Markierung der Buchseiten erleichtert ein rasches Auffinden der gesuchten Produkte. Der Aufbau des Buches ist klar strukturiert. Zahlreiche Bilder und Tabellen erleichtern das Textverständnis. Das Buch schafft einen klaren Überblick über die auf dem Markt angebotene Vielfalt an Wundversorgungsprodukten. Die immer noch zunehmende Fülle an Wundversorgungsprodukten wird regelmäßige, kurzfristige Neuauflagen des Buches notwendig machen, um die derzeitige Aktualität des Kitteltaschenbuches weiterhin zu wahren.

Insgesamt ist es den Autoren gelungen, in kurzer, präziser aber umfassender Form einen Überblick über vorhandene Wundtherapeutika zu geben und damit für den Anwender eine optimale Therapie zu ermöglichen und einen wirtschaftlichen Einsatz der relativ teuren Produktgruppe zu gewährleisten.

Katja Wallenfang-Söhle, Minden 